\title{
'Sterile' Corneal Infiltrates in Contact Lens Wearers
}

\author{
A. K. BATES, ${ }^{1}$ R. J. MORRIS,${ }^{1}$ F. STAPLETON,${ }^{3}$ D. C. MINASSIAN,${ }^{2}$ J. K. G. DART ${ }^{2}$ \\ London
}

\begin{abstract}
Summary
Ninety four patients with 'sterile' keratitis presenting consecutively over a nine month period to the Accident and Emergency Department of Moorfields Eye Hospital were studied. This condition was found to account for $0.49 \%$ of all new casualties. A significant association was found in these patients, compared with controls, with contact lens hygiene, particularly for daily wear soft contact lenses, and contact lens case contamination by bacteria suggesting that these may be important factors in the aetiology of 'sterile' keratitis. Compared to gas permeable hard contact lenses the relative risk of developing 'sterile' keratitis in our patients was found to be 2.3 times higher with extended wear soft contact lenses, 1.56 times higher with daily wear soft contact lenses and 0.509 with polymethylmethacrylate lenses (test of trend p-value $<0.05)$. The results indicate that 'sterile' corneal infiltrates are related to contact lens hygiene and in part to contact lens case contamination by bacteria and also to the type of lens worn.
\end{abstract}

Complications from contact lenses have increased in this country as contact lens wear has become popular, in recent studies at this hospital contact lens wearers accounted for $2.5 \%$ of all new attendances to the Casualty Department ${ }^{1}$ and figures as high as $10 \%$ have been reported. ${ }^{2}$

Suppurative keratitis is the most serious complication associated with contact lens wear. The spectrum of suppurative keratitis extends from the small infiltrate with or without an epithelial defect to the large necrotic corneal abscess, with hypopyon, of fulminating microbial keratitis. Corneal infiltrates may be 'sterile' or microbial, we have used the term 'sterile' to describe non-progressive keratitis because of the uncertain pathogenesis of these lesions some of which may be sterile and others early or spontaneously resolving microbial infiltrates. The distinction between the two in the early stages is difficult on clinical grounds ${ }^{3}$ and is of considerable importance since the latter constitute a serious and potentially sight threatening complication. A group of patients were studied who on clinical grounds had 'sterile' corneal infiltrates. We performed a prospective study to establish the number of patients affected amongst casualty attenders, clinical features of the lesions, the role of contact lens hygiene and contact lens case contamination in the pathogenesis of these lesions, and the relative risk of developing sterile keratitis for the different lens types.

\section{Patients and Methods}

All new patients with a current history of contact lens wear who attended Moorfields Eye Hospital from 21 April 1989 for a nine month period with a clinical diagnosis of 'sterile' keratitis were included in the study.

Inclusion criteria for patients with 'sterile' keratitis were discomfort rather than pain, small lesions with limited or no epithelial

From: Moorfields Eye Hospital, ${ }^{1}$ Institute of Ophthalmology, University of London, ${ }^{2}$ and Department of Optometry and Visual Science, City University ${ }^{3}$

Correspondence to: Mr. AK Bates, Moorfields Eye Hospital, City Road, London EC1V 2PD 
involvement and mild or no anterior chamber reaction. Exclusion criteria were painful lesions with well defined epithelial defects and anterior chamber activity. Patients who were already on treatment were also excluded.

The duration and nature of symptoms prior to attendance was recorded. Clinical features of the corneal infiltrates on slitlamp examination were documented; including site, size and number of infiltrates, area of corneal staining and degree of anterior chamber activity. Infiltrates were considered to be peripheral if any part of them was within $2 \mathrm{~mm}$ of the limbus (Fig. 1), others were described as central (Fig. 2). Arcuate infiltrates were defined as those peripheral lesions which extended over more than one clock hour (Fig. 3).

Patients were followed until symptoms resolved, the infiltrate was epithelialised and reduced in size. The time from presentation to resolution was noted.

Patients were treated with topical G. chloramphenicol $0.5 \%$ (preservative: phenylmercuric acetate $0.002 \%$ ) or G. gentamicin $0.3 \%$ (preservative: benzalkonium chloride $0.02 \%$ ) either four, six or twelve times a day with or without topical G. prednisolone $0.3 \%$ (pre- servative: benzalkonium chloride $0.01 \%$ ) four times a day.

\section{Assessment of hygiene}

Patients completed a questionnaire which included details of the indication for contact lens wear, the type and age of the lens, the cleaning and disinfection regimes used and the pattern of lens wear. In order to evaluate the degree of lens care, each patient was given a hygiene score based on the frequency of lens cleaning and disinfection, and the frequency of use of enzyme tablets. The scores ranged from 0 (poor) to 18 (good). A maximum score of 18 for daily lens wear users was achieved with daily cleaning (7), daily disinfection (7) and weekly use of enzyme tablets (4). To allow extended wear lens users to be compared directly with other wearers, the hygiene scores were based on the level of cleaning and disinfection which occurred each time the lenses were removed. These data were compared with that of a control group of contact lens wearers presenting to the casualty department over the same period of time as the patients with sterile infiltrates, but without keratitis. Hygiene scores for patients with sterile corneal infiltrates and controls were

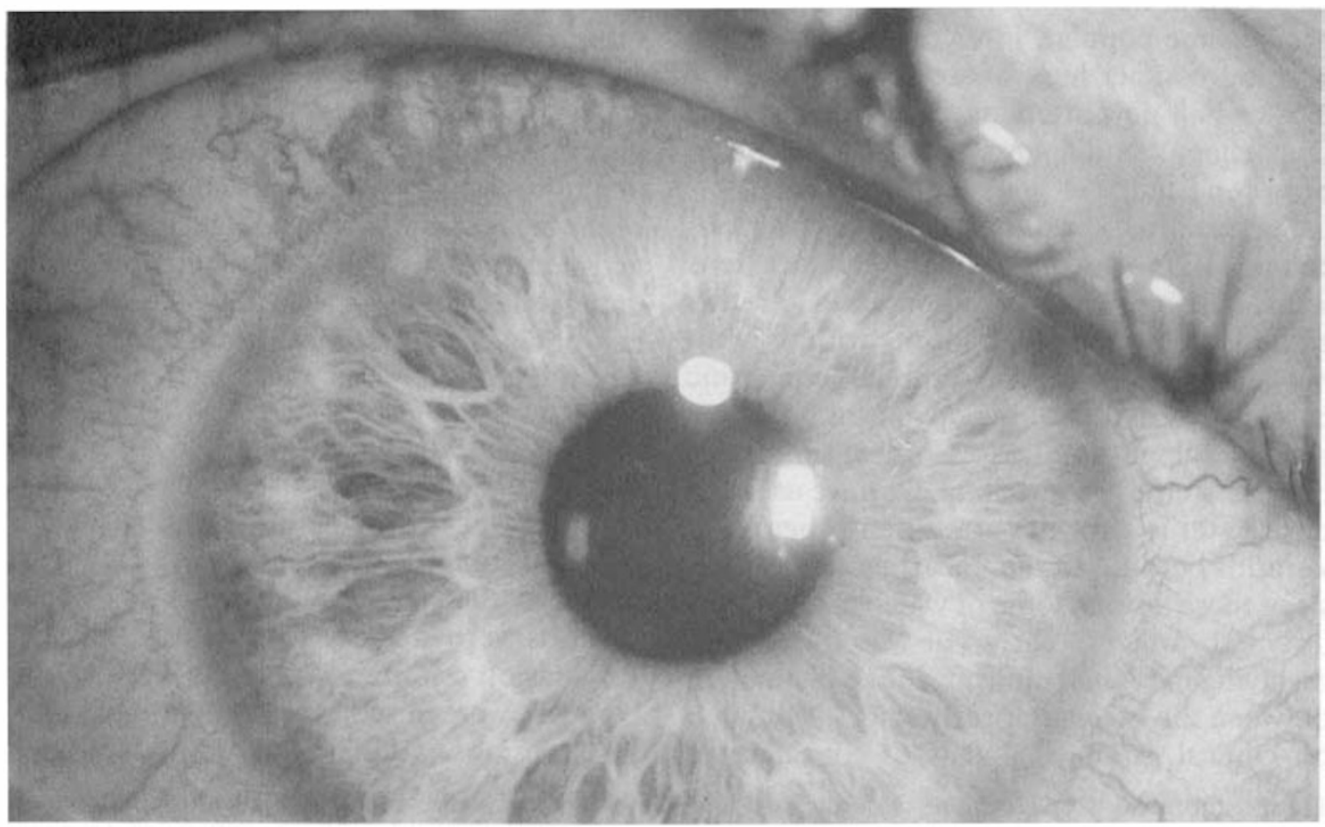

Fig. 1. Peripheral corneal infiltrate. 


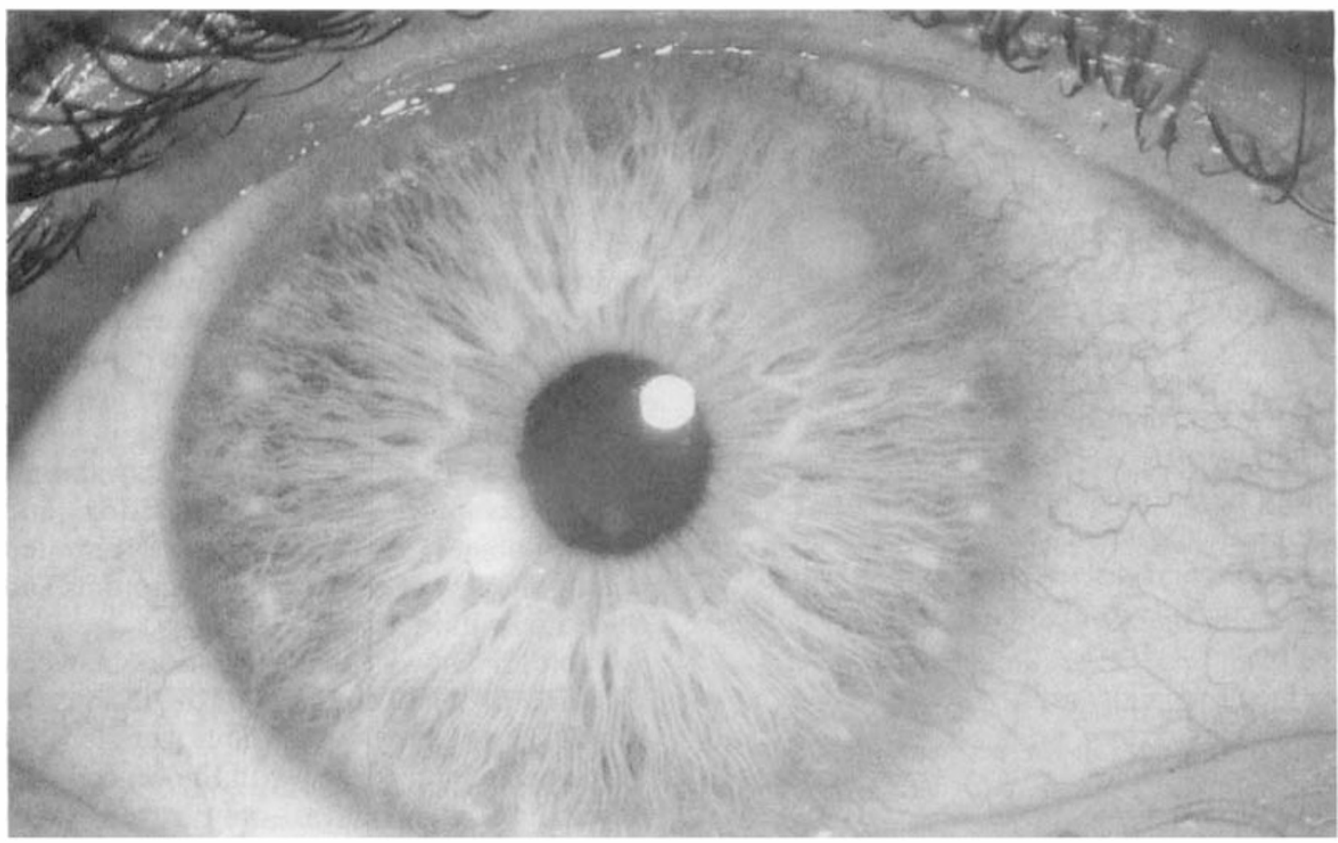

Fig. 2. Central corneal infiltrate.

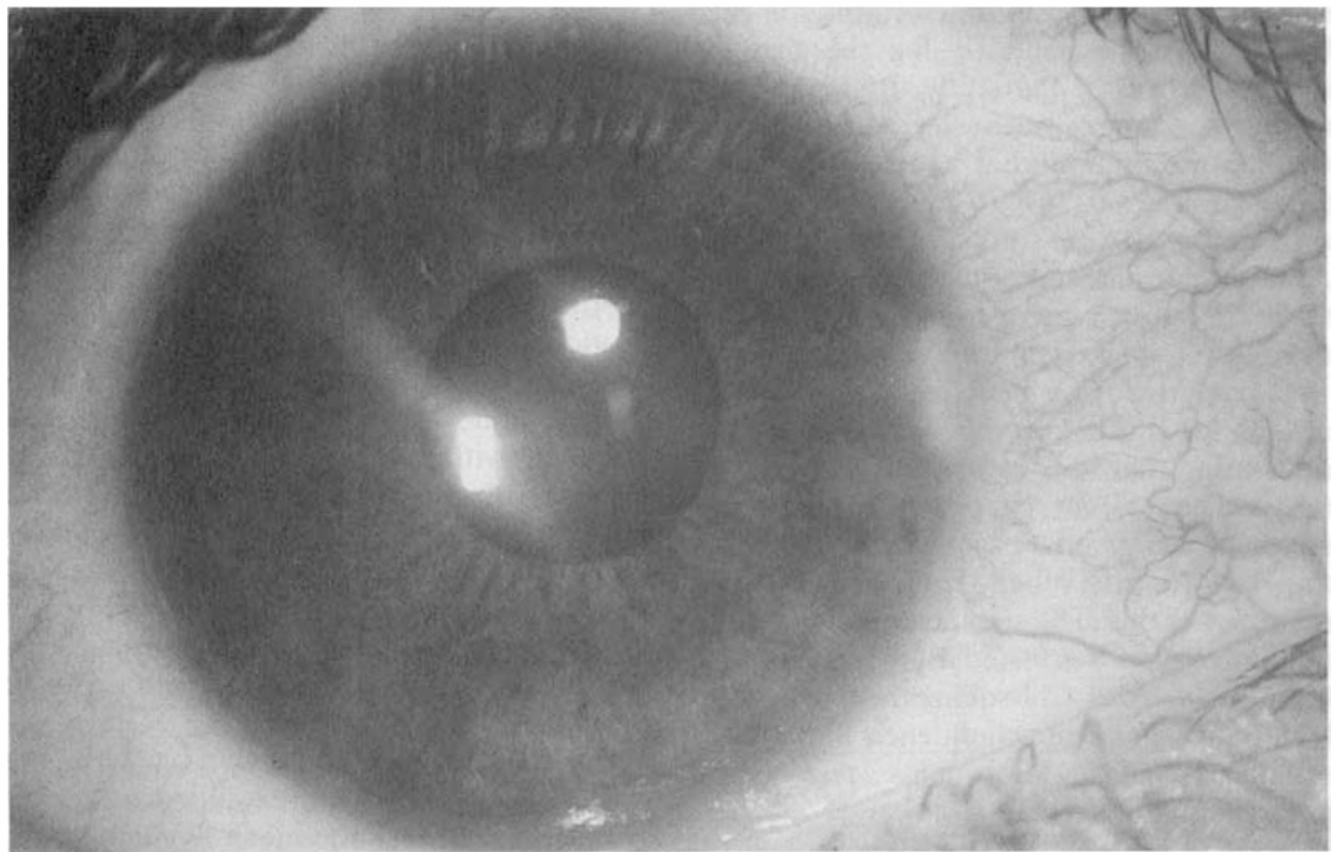

Fig. 3. Arcuate corneal infiltrate. 
compared for each different type of contact lens.

\section{Lens case bacteriology}

Where possible patient's contact lens cases were swabbed and solutions were collected for microbial analysis. Contact lens cases and solutions were stirred with a swab and culture medium innoculated directly. The culture media used were blood agar, Saboraud's agar, McConkey bile salt agar, pseudomonas selective agar (Oxoid CFC), and liquid media (thioglycolate, Robertson's cooked meat and brain heart infusion). The results of bacteriological culture of the patient's contact lens solutions and cases were compared with those of a control group of contact lens wearers without keratitis.

\section{Statistical analysis}

The differences in the proportion of keratitis patients and controls with bacterial contamination of their lens cases was analysed using the Chi square test.

The overall differences in the hygiene scores between the different groups of wearers were analysed using a multifactor analysis of variance (multiway ANOVA). The relative risk of developing 'sterile' keratitis for each lens type was calculated for the first six months period of the study. Rigid gas permeable hard lenses were selected as the referent because pilot studies had suggested that the risk was lowest in hard lens users and highest in extended wear soft contact lens wearers. Also gas permeable hard lenses are assumed to be the safest type of hard lens because of their increased oxygen transmission.

The relative risk associated with each type of lens for sterile keratitis was estimated by calculating the odds ratio from contingency tables. The referent, gas permeable hard contact lens, was given a baseline risk of 1.0 and the risks for the other lenses calculated as multiples of this. The significance of the trend in relative risks was tested using the extended Mantel-Haenszel Chi-square test of trend. ${ }^{4}$ Ninety-five per cent confidence limits were estimated using Miettinen's test based procedure. $^{5}$

\section{Results}

During the period of the study 19,281 new patients attended the Casualty Department $1,125(5.8 \%)$ of whom were contact lens wearers. Ninety-four patients had 'sterile' keratitis which accounted for $0.49 \%$ of all new casualties, $8.4 \%$ of all contact lens wearers presenting to the emergency department and $12.5 \%$ of all lens related problems. Eightyeight patients presented on a single occasion, five twice and one on three occasions. Twentytwo patients were lost to follow-up before resolution of the lesions. Table I shows age, sex, lens type and indication for lens wear.

The most common presenting symptoms were discomfort, redness, watering and photophobia. Forty ( $43 \%)$ patients presented within one day of the onset of symptoms and $81(86 \%)$ within a week.

Table II shows the distribution between eyes and the characteristics of the lesions. In $87(92 \%)$ patients the infiltrates were located in the peripheral cornea with the central cornea being involved in only seven $(8 \%)$ patients: arcuate infiltrates were seen in 13 $(14 \%)$ patients. There was no predilection for any quadrant of the cornea. Fifty one $(54 \%)$ patients had a solitary corneal infiltrate and 43 (46\%) multiple corneal infiltrates.

An epithelial defect was present in 46 cases but was limited to a superficial punctate keratitis or a defect smaller than the lesion itself; in the remaining cases there was no breach of the epithelium. Eighty-three cases had no anterior chamber reaction and in eleven there was minimal anterior chamber activity.

Forty-eight patients were treated with antibiotics alone, 41 with a combination of antibiotics and steroids and five patients received no treatment. In the group treated with antibiotics alone $31(65 \%)$ of the lesions had resolved within a week and in the group treated with antibiotics and steroids $25(61 \%)$ had resolved within a week. In $79(84 \%)$ cases complete resolution occurred without residual corneal scarring and although in 15 $(16 \%)$ there was some residual corneal scarring in no case was there any reduction of visual acuity.

Table III shows an analysis of hygiene scores. Apart from the extended wear soft contact lens group, the mean hygiene scores were consistently lower in the keratitis cases compared to controls. The results of a multi- 
Table I Study patients: age, sex, lens type and indication for lens wear

\begin{tabular}{llrr}
\hline & & No & $(\%)$ \\
\hline Age & & & \\
& Range 19-53 yrs & & \\
& Mean 28.98 yrs & & \\
Sex & Male & 36 & $(38.3)$ \\
& Female & 58 & $(61.7)$ \\
$\quad$ Total & 94 & \\
Lens Type & 79 & $(84.0)$ \\
$\quad$ DW-SCL & 15 & $(20.0)$ \\
& EW-SCL \\
& GP-HCL & 11 & $(11.7)$ \\
$\quad$ PMMA-HCL & 4 & $(4.3)$ \\
Indication for lens wear & & \\
$\quad$ Myopia & 88 & $(93.6)$ \\
$\quad$ Hypermetropia & 5 & $(4.3)$ \\
$\quad$ Aphakia & 1 & $(1.1)$ \\
\hline
\end{tabular}

${ }^{a}$ DW-SCL: Daily wear soft contact lens.

${ }^{b}$ EW-SCL: Extended wear soft contact lens.

${ }^{\mathrm{c}} \mathrm{GP}-\mathrm{HCL}$ : Gas permeable hard contact lens.

${ }^{d}$ PMMA-HCL: Polymethylmethacrylate hard contact lens.

Table II Distribution and characteristics of lesions

\begin{tabular}{|c|c|c|}
\hline & No & $(\%)$ \\
\hline \multicolumn{3}{|l|}{ Side } \\
\hline Right eye & 43 & (46) \\
\hline Left eye & 39 & (41) \\
\hline Both eyes & 12 & (13) \\
\hline \multicolumn{3}{|l|}{ Site } \\
\hline Peripheral & 87 & (92) \\
\hline Central & 7 & $(8)$ \\
\hline Arcuate & 13 & (14) \\
\hline \multicolumn{3}{|l|}{ Number } \\
\hline Single & 51 & (54) \\
\hline Multiple & 43 & (46) \\
\hline \multicolumn{3}{|l|}{ Epithelial defect } \\
\hline Present & 46 & (49) \\
\hline Absent & 48 & (51) \\
\hline \multicolumn{3}{|c|}{ Anterior cham ber activity } \\
\hline Present & 11 & (12) \\
\hline Absent & 83 & (88) \\
\hline
\end{tabular}

factor analysis of variance are shown in Table IV and indicate that the keratitis cases had significantly lower hygiene scores compared to controls in the three lens-type groups $(F=5.34, p=0.02)$. Homogenicity of this phenomenon across all three groups of lens types is indicated by the absence of a significant 2-factor interaction $(p=0.79)$, which suggests that the differences in hygiene scores between keratitis cases and controls, are inde- pendent of the three lens types. Further analysis of variance including the extended wear soft contact lens group, showed a significant association between lens type and hygiene score, manifesting mainly as lower hygiene scores in the extended wear group compared to wearers of other lens types $(\mathrm{F}=4.41$, $\mathrm{p}=0.005$ ).

The results of microbial cultures of contact lens cases are shown in Table V. In the study group 15 cases were obtained for culture and in the control group 43 cases. Ten $(66.7 \%)$ cases in the study group were found to be contaminated by bacteria and $16(37.2 \%)$ in the control group. These differences were significant $\mathrm{p}=0.048$.

The relative risk of developing sterile keratitis for the different lenses are shown in Table VI. Compared to gas permeable hard contact lenses this was highest for soft contact lenses particularly for extended wear soft contact lenses at 2.33 and lowest with polymethylmethacrylate hard contact lenses at 0.509 . However, the trend of increasing risk for soft contact lenses was significant $(\mathrm{p}<0.05)$.

\section{Discussion}

Suppurative keratitis is a non-specific descriptive term for corneal infiltrates of any cause. Clinically three main types of infiltrates may be identified. (1) Small (less than $1 \mathrm{~mm}$ ) peripheral infiltrates with no epithelial defect

Table III Analysis of contact lens hygiene scores

\begin{tabular}{|c|c|c|c|}
\hline $\begin{array}{l}\text { Contact } \\
\text { Lens type }\end{array}$ & $\begin{array}{l}\text { Number } \\
\text { of patients }\end{array}$ & $\begin{array}{c}\text { Mean } \\
\text { hygiene score }\end{array}$ & $\begin{array}{c}\text { Standard } \\
\text { error }\end{array}$ \\
\hline \multicolumn{4}{|l|}{$G P-H C L^{a}$} \\
\hline Cases & 4 & 9.00 & 1.68 \\
\hline Controls & 25 & 10.64 & 0.70 \\
\hline \multicolumn{4}{|c|}{$P M M A-H C L^{b}$} \\
\hline Cases & 11 & 12.18 & 1.00 \\
\hline Controls & 39 & 12.70 & 0.72 \\
\hline \multicolumn{4}{|l|}{$D W-S C L^{c}$} \\
\hline Cases & 64 & 10.48 & 0.56 \\
\hline Controls & 82 & 12.10 & 0.47 \\
\hline \multicolumn{4}{|l|}{$E W-S C L^{d}$} \\
\hline Cases & 15 & 9.27 & 1.30 \\
\hline Controls & 8 & 7.13 & 1.42 \\
\hline Total & 248 & & \\
\hline
\end{tabular}

${ }^{a}$ GP-HCL: Gas permeable hard contact lens.

${ }^{b}$ PMMA-HCL: Polymethylmethacrylate hard contact lens.

${ }^{\mathrm{c}}$ DW-SCL: Daily wear soft contact lens.

${ }^{\mathrm{s}}$ EW-SCL: Extended wear soft contact lens. 
Table IV Multifactor analysis of variance-comparison of mean hygiene scores of cases and controls in GP$H C L^{a}, P M M A-H C L^{b}$ and $D W-S C L^{c}$ (summary of results)

\begin{tabular}{|c|c|c|c|c|}
\hline Source & $\begin{array}{l}\text { Degrees of } \\
\text { freedom }\end{array}$ & $\begin{array}{l}\text { Mean sum } \\
\text { of squares }\end{array}$ & F-ratio & $P$-value \\
\hline \multicolumn{5}{|l|}{ Keratitis } \\
\hline (Case/Control) & 1 & 95.208 & 5.35 & 0.02 \\
\hline Lens type & 2 & 47.775 & 2.69 & 0.07 \\
\hline \multicolumn{5}{|l|}{ 2-Factor interaction } \\
\hline lens type $\times$ Keratitis & 2 & 4.218 & 0.237 & 0.79 \\
\hline Residuals & 219 & 17.781 & & \\
\hline Totals & 224 & & & \\
\hline
\end{tabular}

${ }^{a}$ GP-HCL: Gas permeable hard contact lens.

${ }^{\mathrm{b}}$ PMMA-HCL: Polymethylmethacrylate hard contact lens.

${ }^{c}$ DW-SCL: Daily wear soft contact lens.

Table $\mathbf{V}$ Contact lens case contamination by bacteria

\begin{tabular}{|c|c|c|c|c|c|}
\hline & \multicolumn{2}{|c|}{ Study group } & \multicolumn{2}{|c|}{ Controls } & \multirow[t]{2}{*}{$p$ value $\left(\chi^{2}\right)$} \\
\hline & No & $(\%)$ & No & $(\%)$ & \\
\hline Negative cultures & 5 & & 27 & & \\
\hline Positive cultures & 10 & $(66.7 \%)$ & 16 & $(37.2 \%)$ & $0.048(3.90)$ \\
\hline \multicolumn{6}{|l|}{ Non-lactose fermenting } \\
\hline Gram negative organisms (NLF GNR) & 5 & & 12 & & \\
\hline Serratia marcescens & 3 & & 0 & & \\
\hline Pseudomonas aerogenosa & 1 & & 0 & & \\
\hline Enterobacteria & 1 & & 1 & & \\
\hline Mixed cultures & 0 & & $3 *$ & & \\
\hline
\end{tabular}

* 2 mixed cultures of staphylococcus, enterobacteria and micrococci.

1 mixed culture of staphylococcus aureus and NLF GNR's.

and no anterior chamber reaction, which are not associated with pain or discharge. This group is thought to be sterile (2) Larger (greater than $2 \mathrm{~mm}$ ) infiltrates with epithelial defects and anterior chamber activity, which are associated with pain and discharge. This group is thought to be infected even though in some cases the organism cannot be cultured.
(3) Between these groups is a spectrum of lesions which may or may not be infected.

Infiltrates which are central, associated with pain, discharge, epithelial staining or anterior chamber reaction suggest infection. ${ }^{3}$ Sterile infiltrates, less commonly associated with these features, are usually smaller and may be multiple or arcuate. However, the dis-

Table VI The relative risk of different contact lenses for "sterile" keratitis

\begin{tabular}{lcccc}
\hline Type of lens & Cases & Controls & Relative risk & 95\% confidence limits \\
\hline GP-HCL $^{\mathrm{a}}$ & 9 & 126 & 1.0 (referent) & $0.2-1.7$ \\
PMMA-HCL $^{\mathrm{b}}$ & 4 & 110 & 0.509 & $0.8-3.2$ \\
DW-SCL $^{\mathrm{c}}$ & 50 & 448 & 1.56 & $1.0-5.5$ \\
EW-SCL $^{\mathrm{d}}$ & 14 & 84 & 2.33 & . \\
\hline
\end{tabular}

Extended Mantel-Haenszel Test of Trend $\chi^{2}=6.005$

$\mathrm{P}$-value $<0.005$

${ }^{\text {a }}$ GP HCL: Gas permeable hard contact lens.

${ }^{b}$ PMMA-HCL: Polymethylmethacrylate hard contact lens.

${ }^{c}$ DW-SCL: Daily wear soft contact lens.

${ }^{\mathrm{d}}$ EW-SCL: Extended wear soft contact lens. 
tinction between sterile and infected corneal infiltrates may be difficult on clinical grounds alone and since the consequences of untreated microbial keratitis may be devastating it is prudent to treat lesions as infected if any doubt exists.

Patients included in this study had on clinical grounds a 'sterile' keratitis with minimal symptoms, little discharge, small corneal lesions with limited epithelial involvement and little or no anterior chamber activity. Ninety-four patients presented with these features and accounted for $0.49 \%$ of all new casualties. Although it has been well documented that contact lens wear is a major predisposing factor for microbial keratitis, ${ }^{6-9}$ we are not aware of other reports showing this high incidence of 'sterile' keratitis in a contact lens wearing population.

In the majority of patients infiltrates were peripheral $(92.3 \%)$ with arcuate defects accounting for $13.8 \%$ of cases. In $51 \%$ of cases there was no epithelial defect and in the remainder there was some breach of the epithelial surface. In only eleven cases was there any anterior chamber activity. There was no difference in the time to resolution of the lesions treated with antibiotics alone or with steroids and antibiotics. Due to the design of the study, however, it was not possible to determine the relative efficacy of different treatments in the resolution of the lesions. None of the patients progressed to fulminating microbial keratitis despite the potential difficulty in distinguishing between sterile and microbial keratitis in some patients. Two possible explanations of this are: firstly the aetiology of sterile infiltrates is different and secondly it is possible that some of our cases did have an early infection but that early presentation and prompt treatment with topical antibiotics prevented progression to fulminating keratitis.

The aetiology of 'sterile' keratitis is not well established although several theories have been proposed. It is likely that it is an immunologically mediated reaction to the lens material itself ${ }^{10}$ or a toxicity reaction to the solutions used in cleaning and sterilisation. ${ }^{11-13}$ Alternatively bacteria or toxins adherent to the surface of the lens may present an antigenic load. ${ }^{14}$ In some cases the infiltrates may represent a hypersensitivity reaction to sta- phylococci. ${ }^{15}$ Other cases may represent a spontaneously resolving infection or an early infection.

Little information is available on the importance of various risk factors relevant to these theories. ${ }^{16}$ The relationship of lens hygiene, microbial contamination of the lens cases, lens type and the pattern of contact lens wear has been investigated in this study.

We found a statistically significant difference in hygiene scores between those patients with 'sterile' corneal infiltrates and controls. When each lens type is analysed separately the difference is most marked in the daily wear soft contact lens group. However, in the extended wear soft contact lens group there is no significant difference and we believe that this may be related to the small numbers of controls in this group. The results also show an association between lens types and hygiene scores with significantly lower hygiene scores in the extended wear group compared to wearers of other lenses. These findings suggest that this complication of contact lens wear is related to the degree of contact lens hygiene practised by patients, but because the association is relatively weak it is likely to be one of several factors. To our knowledge this is the first time that such an association has been shown. This evidence is supported by the finding of a significant difference in the microbial contamination of lens cases between the study group and controls. However, it is notable that $37.2 \%$ of controls had contaminated lenses, a finding that has also been described in asymptomatic contact lens wearers. ${ }^{17,18}$

The relative risks of different contact lens types for all complications of contact lens wear has been reported and showed higher risks for soft contact lens wear. ${ }^{2}$ Using polymethylmethacrylate lenses as the referent the risk (with 95\% confidence limits) for daily wear soft contact lenses was $2.0(1.1-3.3)$ and for extended wear lenses 6.8 (1.8-25.6). Also several retrospective studies have shown that contact lenses are likely to be a major predisposing factor in microbial keratitis. ${ }^{6-9}$ To our knowledge the relative risks of these different lenses for sterile keratitis has not been reported. This study shows that there was a significantly increased trend of risk associated with soft contact lens wear, being greatest for extended wear soft contact lens wear: poly- 
methylmethacrylate hard contact lenses had the lowest risk. These differences may be the results of the interactions of different lens materials with the ocular surface, antigens and bacteria, different preservatives in hard and soft contact lens solutions and different patterns of wearing time.

Our results suggest that contact lens hygiene, lens case contamination and the type of lens worn are significant factors in the aetiology of 'sterile' infiltrates. Patients considering wearing contact lenses should be advised of the increased risks for complications associated with soft contact lenses and particularly extended wear contact lenses. Those presenting with corneal infiltrates should be counselled by the clinician that the risk of further episodes is related to contact lens hygiene, contact lens case contamination and the type of lens worn and that improved lens hygiene may reduce the risk of further episodes.

We would like to thank the casualty registrars who participated in the recruitment of patients. Mr. M Matheson and Mr. J Peacock performed the microbiological studies. F Stapleton is funded by a grant from the British Council of Optometrists. The study was supported by grants from Moorfields Eye Hospital LOR 89/10, The Association of Contact Lens Manufacturers and the British Council for the Prevention of Blindness.

\section{References}

${ }^{1}$ Barry PJ and Ruben M: Contact lens injuries. An analysis of 217 consecutive cases presenting to Moorfields casualty department. Cont act Lens J 1980, 9: 959-60.

${ }^{2}$ Franks WA, Adams GGW, Dart JKG, Minassian D: Relative risks of different types of contact lenses. Br Med J 1988, 297: 524-5.

${ }^{3}$ Stein RM, Clinch TE, Cohen EJ, Genvert GI, Arentsen JJ, Liabson PR: Infected vs sterile corneal infiltrates in contact lens wearers. Am J Opthalmol 1988, 105: 632-6.
${ }^{4}$ Mantel N: Chi-square tests with one degree of freedom: extensions of the Mantel-Haenszel procedure. J Am Stat Ass 1963, 58: 690-700.

${ }^{5}$ Mienttinen OS: Simple interval-estimation of risk ratio. Am J Epidemiol 1974, 100: 515-6.

${ }^{6}$ Alfonso E, Mandlbaum S, Fox MJ, Forster RK: Ulcerative keratitis associated with contact lens wear. Am J Ophthalmol 1986, 101: 429-33.

${ }^{7}$ Ormerod LD and Smith RE: Contact lens associated microbial keratitis. Arch Ophthalmol 1986, 104: 79-83.

${ }^{8}$ Galentine PG, Cohen EJ, Laibson PR, Adams CP, Michaud R, Arentsen JJ: Corneal ulcers associated with contact lens wear. Arch Ophthalmol 1984, 102: 891-4.

${ }^{9}$ Dart JKG: Predisposing factors in microbial keratitis: the significance of contact lens wear. $\mathrm{Br} \mathrm{J}$ Ophthalmol 1988, 72: 926-30.

${ }^{10}$ Bernstein HN and Kemp MA: An unusual keratoconjunctivitis occurring after longtime wearing of the AO Softcon (formerly Griffin or Bionite) hydrophilic contact lens. Ann Ophthalmol 1975, 7: 97-106.

${ }^{11}$ Binder PS, Rasmussen DM, Gordon M: Keratoconjunctivitis and soft contact lens solutions. Arch Ophthalmol 1981, 99: 87-90.

${ }^{12}$ Mondino BJ and Groden LR: Conjunctival hyperaemia and corneal infiltrates with chemically disinfected contact lens solutions. Arch Opthalmol 1980, 98: 1767-70.

${ }^{13}$ Wilson LA, McNatt J, Reitschel R: Delayed hypersensitivity to thiomersal in soft contact lens wearers. Opthalmology 1981, 88: 804-9.

${ }^{14}$ Phillips AJ, Badenoch PR, Grutzmacher R, Roussel TJ: Microbial contamination of extended-wear contact lenses: An investigation of endotoxin as a cause of the acute ocular inflammation reaction. Int ernational Eyecare 1986, 9: 469-75.

${ }^{15}$ Smolin G, Okumoto M, Nozik RA: The microbial flora in extended-wear soft contact-lens wearers. Am J Ophthalmol 1979, 88: 543-7.

${ }^{16}$ Schein O, Hibberd P, Kenyon KR: Contact lens complications: incidental or epidemic? Am J Opthalmol 1986, 102: 116-7.

${ }^{17}$ Pitts RE, Krachmer JH: Evaluation of soft contact lens contamination in the home environment. Arch Ophthalmol 1979, 97: 470-2.

${ }^{18}$ Donzis P B, Mondino BJ, Weissman BW, Bruckner DA: Microbial contamination of contact lens care systems. Am J Ophthalmol 1987, 104: 325-33. 